Acknowledgements: The authors thank the children who participated and their families, and the primary school "Ecole de Brabois", Vandoeuvre, France.

\title{
References
}

1 Cauberghs M, Van de Woestijne KP. Mechanical properties of the upper airway. J Appl Physiol Respir Environ Exerc Physiol 1983; 55: 335-342.

2 Peslin R, Ying Y, Gallina C, et al. Within-breath variations of forced oscillation resistance in healthy subjects. Eur Respir J 1992; 5: 86-92.

3 Brancatisano T, Collett PW, Engel LA. Respiratory movements of the vocal cords. J Appl Physiol Respir Environ Exerc Physiol 1983; 54: 1269-1276.

4 Schweitzer C, Chone C, Marchal F. Influence of data filtering on reliability of respiratory impedance and derived parameters in children. Pediatr Pulmonol 2003; 36: 502-508.

5 Baier H, Wanner A, Zarzecki S, et al. Relationships among glottis opening, respiratory flow, and upper airway resistance in humans. J Appl Physiol Respir Environ Exerc Physiol 1977; 43: 603-611.

6 Collett PW, Brancatisano T, Engel LA. Changes in the glottic aperture during bronchial asthma. Am Rev Respir Dis 1983; 128: 719-723.

7 Higenbottam T. Narrowing of glottis opening in humans associated with experimentally induced bronchoconstriction. J Appl Physiol Respir Environ Exerc Physiol 1980; 49: 403-407.

$8 \quad$ Higenbottam T, Payne J. Glottis narrowing in lung disease. Am Rev Respir Dis 1982; 125: 746-750.

9 Stanojevic S, Wade A, Stocks J, et al. Reference ranges for spirometry across all ages: a new approach. Am J Respir Crit Care Med 2008; 177: 253-260.

10 Peslin R, Felicio da Silva J, Duvivier C, et al. Respiratory mechanics studied by forced oscillations during artificial ventilation. Eur Respir J 1993; 6: 772-784.

11 Gauthier R, Beyaert C, Feillet F, et al. Respiratory oscillation mechanics in infants with bronchiolitis during mechanical ventilation. Pediatr Pulmonol 1998; 25: 18-31.

12 Oostveen E, MacLeod D, Lorino $\mathrm{H}$, et al. The forced oscillation technique in clinical practice: methodology, recommendations and future developments. Eur Respir J 2003; 22: 1026-1041.

13 Calogero C, Simpson SJ, Lombardi E, et al. Respiratory impedance and bronchodilator responsiveness in healthy children aged 2-13 years. Pediatr Pulmonol 2013; 48: 707-715.

14 Gobbi A, Pellegrino R, Gulotta C, et al. Short-term variability in respiratory impedance and effect of deep breath in asthmatic and healthy subjects with airway smooth muscle activation and unloading. J Appl Physiol (1985) 2013; 115: 708-715.

15 Peslin R, Farré R, Rotger M, et al. Effect of expiratory flow limitation on respiratory mechanical impedance: a model study. J Appl Physiol (1985) 1996; 81: 2399-2406.

\section{Job strain and COPD exacerbations: an individual-participant meta-analysis}

To the Editor:

Chronic obstructive pulmonary disease (COPD) is a major cause of mortality and disability worldwide [1]. The clinical course of COPD is characterised by exacerbations, which can be minor and manageable at home or in primary care, or severe, leading to hospitalisation or even death. Known causes of exacerbations include tobacco smoke, air pollution, dusts and fumes, and respiratory infections $[1,2]$. One less well understood risk factor is stress, which could plausibly lead to COPD exacerbations as it can trigger inflammation [3,4] and is associated with increased smoking [5], which are both implicated in COPD pathology [2]. Work is an important source of stress in the age groups in which COPD is typically diagnosed $[1,6]$. However, we are not aware of previous investigations of work-related stress and the risk of COPD exacerbations.

In this study, we examined the associations between job strain (the most widely studied conceptualisation of work-related stress) and severe COPD exacerbations using individual-level data from 10 prospective cohort studies from the Individual Participant Data Meta-analysis in Working Populations (IPD-Work) Consortium [7]. Job strain is defined as a combination of high demands (excessive amounts of work) and low control (having little influence on what tasks to do and how to carry them out) at work.

We ascertained job strain from the participants' responses to questions on demands and control aspects of their work at study baseline. The responses were scored and for each participant, and mean scores were calculated for job-demand items and job-control items. Based on these, participants' job demands and job control were defined as high or low. A combination of high demands (a job demand score above the studyspecific median) and low control (job control score below the study-specific median) was defined as high 
strain and all other combinations as no strain. Job strain was validated and harmonised across the studies [7] and modelled as a binary exposure (high strain versus no strain).

Severe COPD exacerbations were ascertained from national hospitalisation and death registers and defined as International Classification of Diseases version 9 codes 491, 492 and 496, or version 10 codes J41, J42, J43 and J44. Our analyses were adjusted for harmonised age, sex, socioeconomic position, body mass index (BMI), tobacco smoking and alcohol intake.

We modelled the study-specific associations between job strain and COPD exacerbations using Cox regression. The Schoenfeld test suggested the proportional hazards assumption to be valid. Study-specific estimates were pooled in random effects meta-analyses and heterogeneity among these quantified using the $\mathrm{I}^{2}$-statistic. All statistical analyses were conducted using Stata 11 (Stata Corporation Ltd, College Station, TX, USA), apart from study-specific analyses in the Danish studies, which were conducted using SAS 9.2 (SAS Institute Inc., Cary, NC, USA).

Our analyses were based on 92428 males and females (median age at baseline: 42 years), of whom 451 experienced a COPD exacerbation leading to hospitalisation $(n=431)$ or death $(n=20)$ during the follow-up (table 1). The median length of follow-up was 10 years, ranging from 2 to 23 years, and the mean \pm SD age at first severe COPD exacerbation was $60 \pm 9$ years. Participants with job strain at baseline had a $32 \%$ higher risk of a severe COPD exacerbation (hazard ratio (HR) 1.32, 95\% CI 1.04-1.68) than those with no strain in the age and sex-adjusted analyses (table 1). However, with additional adjustment for BMI, alcohol intake, smoking and socioeconomic position, this association attenuated to the null (HR 1.10, 95\% CI 0.86-1.41).

When we stratified our analyses by baseline smoking status, there was no robust evidence for an association between job strain and the risk of COPD exacerbations among baseline smokers or baseline never-smokers after adjustment for potential confounders. Among baseline ex-smokers, job strain (compared with no strain) was associated with a 2.11-fold risk of a severe exacerbation (95\% CI 1.03-4.31), independently of age, sex, socioeconomic position, BMI and alcohol intake (24539 ex-smokers, including 63 cases of severe COPD exacerbation, 15 among participants with and 48 among those without job strain). All findings were similar in the analyses with the job strain model quadrants (passive job, active job and high strain compared with low strain) as the exposure as well as in sensitivity analyses with all asthma cases excluded.

In our meta-analyses, job strain was not associated with the risk of severe COPD exacerbations, independently of age, sex, socioeconomic position, BMI, smoking and alcohol intake. We found no association between job strain and severe COPD exacerbations among baseline never-smokers or baseline smokers but, among baseline ex-smokers, job strain was associated with an approximately two-fold increased risk of a severe exacerbation. The last finding should be interpreted as hypothesis generating, as it was based on small numbers and could be a chance finding. Baseline ex-smokers with COPD may have a more advanced disease than the COPD cases in the other smoking categories and our findings may also reflect the high risk of exacerbation at advanced stages of COPD (with the disease process causing both stress and exacerbations).

An important strength of our meta-analysis was that we used a large set of prospectively collected individual-level data. Of the 10 studies, five were population-based and another five were workplace-based but included participants from across the socioeconomic range (table 1). Thus, our findings are likely generalisable to the northern European workforce. We used a validated and harmonised exposure, job strain [7], and register-based COPD outcomes, which have good coverage and which are generally not prone to recall or other biases [8-10]. In addition to in-patient data, the hospitalisation registers covered outpatient appointments onwards from the mid-1990s in the Finnish and Danish studies [11] and from 2003 in the UK study [12].

It is a limitation of our study that a single baseline measurement of job strain may have been too imprecise an indicator of the exposure, which may have diluted our association estimates. Further research with repeated measurements of job strain over time would clarify this issue. Another limitation is that we had no data on disease severity or lung function at baseline. Other unmeasured confounders that might have influenced our estimates and should be controlled for in future studies include occupational exposures, concurrent illnesses, social support and physical activity. Finally, as our outcomes were severe COPD exacerbations, our findings are not necessarily generalisable to mild exacerbations.

We adjusted our analyses for smoking and alcohol intake but these could plausibly be on the causal pathway between job strain and COPD exacerbations. However, previous analyses of the IPD-Work Consortium data provided no conclusive evidence for longitudinal associations of job strain with smoking or alcohol intake, though job strain was associated with increasing smoking intensity. Further research would help to 


\begin{tabular}{|c|c|c|c|}
\hline 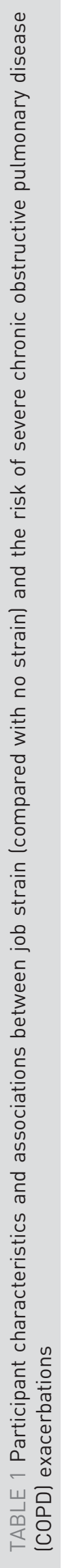 & 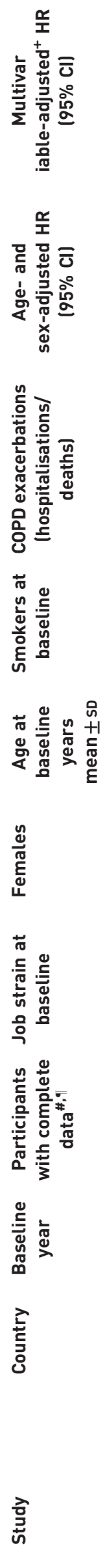 & 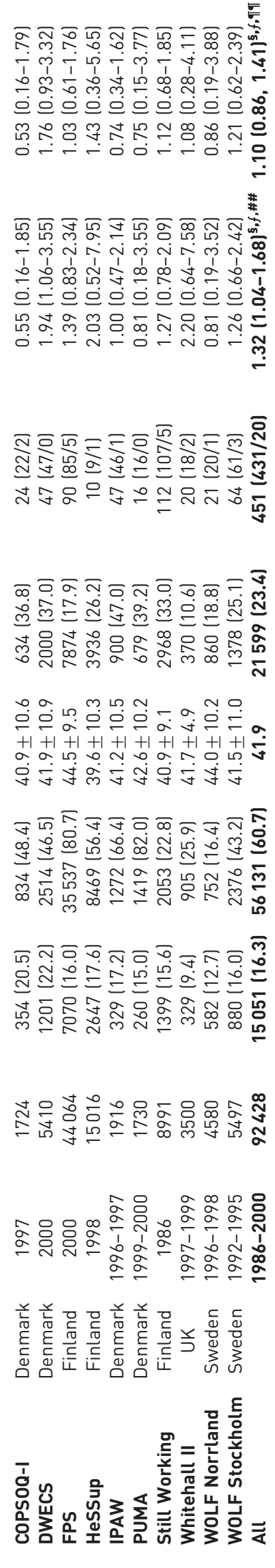 & 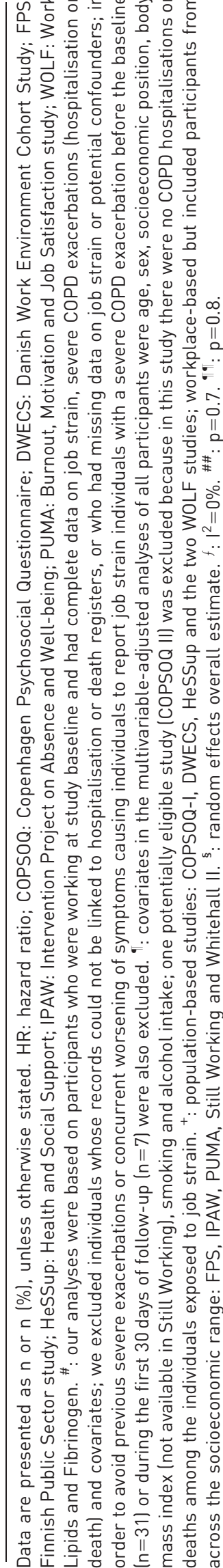 \\
\hline
\end{tabular}


understand the direction of these associations, for if smoking and alcohol were mediators, job strain might be associated with COPD exacerbations via these risk factors.

In conclusion, our meta-analyses suggest that job strain is not associated with severe COPD exacerbations, though imprecise exposure or residual confounding may have influenced our results. This investigation should therefore be considered a hypothesis-generating study to encourage further research in this area. Importantly, associations between repeated measurements of job strain and indicators of COPD severity (e.g. lung function tests) would merit further study.

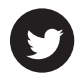
@ERSpublications

Multiple cohort studies do not support job strain being a major independent risk factor for severe COPD exacerbations http://ow.ly/tAyq2

Katriina Heikkilä ${ }^{1}$, Ida E.H. Madsen ${ }^{2}$, Solja T. Nyberg ${ }^{1}$, Eleonor I. Fransson ${ }^{3,4,5}$, Kirsi Ahola ${ }^{1}$, Lars Alfredsson ${ }^{3,6}$, Jakob B. Bjorner ${ }^{2}$, Marianne Borritz ${ }^{7}$, Hermann Burr ${ }^{8}$, Anders Knutsson ${ }^{9}$, Markku Koskenvuo ${ }^{10}$, Aki Koskinen ${ }^{1}$, Martin L. Nielsen ${ }^{7}$, Maria Nordin ${ }^{11}$, Krista Pahkin ${ }^{1}$, Jaana Pentti ${ }^{12}$, Reiner Rugulies ${ }^{2,13}$, Paula Salo ${ }^{12,14}$,

Martin J. Shipley ${ }^{15}$, Sakari B. Suominen ${ }^{16,17,18}$, Töres Theorell ${ }^{5}$, Ari Väänänen ${ }^{1}$, Jussi Vahtera ${ }^{12,17}$, Marianna Virtanen ${ }^{1}$, Peter J.M. Westerholm ${ }^{19}$, G. David Batty ${ }^{15,20}$, Archana Singh-Manoux ${ }^{15,21}$ and Mika Kivimäki ${ }^{1,15}$ for the IPD-Work Consortium

${ }^{1}$ Finnish Institute of Occupational Health, Helsinki, ${ }^{10}$ Dept of Public Health, University of Helsinki, Helsinki, ${ }^{12}$ Finnish Institute of Occupational Health, Turku, ${ }^{14}$ Dept of Psychology, University of Turku, Turku, ${ }^{16}$ Folkhälsan Research Center, Helsinki, and ${ }^{17}$ Dept of Public Health, University of Turku, Turku, Finland. ${ }^{2}$ National Research Centre for the Working Environment, Copenhagen, ${ }^{7}$ Dept of Occupational and Environmental Medicine, Bispebjerg University Hospital, Copenhagen, and ${ }^{13}$ Dept of Public Health and Dept of Psychology, University of Copenhagen, Copenhagen, Denmark. ${ }^{3}$ Institute of Environmental Medicine, Karolinska Institutet, Stockholm, ${ }^{4}$ School of Health Sciences, Jönköping University, Jönköping, ${ }^{5}$ Stress Research Institute, Stockholm University, Stockholm, ${ }^{6}$ Centre for Occupational and Environmental Medicine, Stockhom County Council, Stockhom, ${ }^{9}$ Dept of Health Sciences, Mid Sweden University, Sundsvall, ${ }^{11}$ Dept of Psychology, Umeå University, Umeå, ${ }^{18}$ Nordic School of Public Health, Gothenburg, and ${ }^{19}$ Occupational and Environmental Medicine, Uppsala University, Uppsala, Sweden. ${ }^{8}$ Federal Institute for Occupational Safety and Health (BAuA), Berlin, Germany. ${ }^{15}$ Dept of Epidemiology and Public Health, University College London, London, and ${ }^{20}$ Centre for Cognitive Ageing and Cognitive Epidemiology, University of Edinburgh, Edinburgh, UK.

${ }^{21}$ Inserm U1018, Centre for Research in Epidemiology and Population Health, Villejuif, France.

Correspondence: Katriina Heikkilä, Finnish Institute of Occupational Health, Uimalankatu 1, 33101 Tampere, Finland. E-mail: katriina.heikkila@ttl.fi

Received: Sept 102013 | Accepted after revision: Feb 032014 | First published online: April 022014

Support statement: The IPD-Work Consortium supported by the European Union New OSH ERA research programme (funded by the Finnish Work Environment Fund, the Swedish Research Council for Working Life and Social Research, and the Danish National Research Centre for the Working Environment), the Academy of Finland (132944) and the BUPA Foundation (22094477). M. Kivimäki is supported by the UK Medical Research Council (K013351), the US National Institutes of Health (R01HL036310 and R01AG034454), and a professorial fellowship from the UK Economic and Social Research Council. Details of funding for each constituent study are provided on the study websites. The funding bodies had no role in the study design, data collection or analysis, decision to publish the findings, or preparation of the manuscript.

Conflict of interest: Disclosures can be found alongside the online version of this article at www.erj.ersjournals.com

\section{References}

1 Global Initiative for Chronic Obstructive Lung Disease. Global strategy for the diagnosis, management, and prevention of chronic obstructive pulmonary disease, www.goldcopd.org/uploads/users/files/GOLD_Report_2013_ Feb20.pdf Date last accessed: November 8, 2013. Date last updated: 2013.

2 Bhalla DK, Hirata F, Rishi AK, et al. Cigarette smoke, inflammation, and lung injury: a mechanistic perspective. J Toxicol Environ Health 2009; 12: 45-64.

3 Slavich GM, Way BM, Eisenberger NI, et al. Neural sensitivity to social rejection is associated with inflammatory responses to social stress. Proc Natl Acad Sci USA 2010; 107: 14817-14822.

4 Steptoe A, Hamer M, Chida Y. The effects of acute psychological stress on circulating inflammatory factors in humans: a review and meta-analysis. Brain Behav Immun 2007; 21: 901-912.

5 Heikkilä K, Nyberg ST, Fransson EI, et al. Job strain and tobacco smoking: an individual-participant data metaanalysis of 166130 adults in 15 European studies. PLoS ONE 2012; 7: e35463.

6 Milczarek M, Schneider EE, Rial González E. OSH in Figures: Stress at Work - Facts and Figures, Luxembourg, European Agency for Safety and Health at Work, 2009.

7 Fransson EI, Heikkila K, Nyberg ST, et al. Job strain as a risk factor for leisure-time physical inactivity: an individual-participant meta-analysis of up to 170,000 men and women: the IPD-Work Consortium. Am J Epidemiol 2012; 176: 1078-1089.

8 Inghammar M, Engstrom G, Lofdahl CG, et al. Validation of a COPD diagnosis from the Swedish Inpatient Registry. Scand J Public Health 2012; 40: 773-776.

9 Sund R. Quality of the Finnish Hospital Discharge Register: a systematic review. Scand J Public Health 2012; 40: 505-515. 
10 Thomsen RW, Lange P, Hellquist B, et al. Validity and underrecording of diagnosis of COPD in the Danish National Patient Registry. Respir Med 2011; 105: 1063-1068.

11 Lynge E, Sandegaard JL, Rebolj M. The Danish National Patient Register. Scand J Public Health 2011; 39: 30-33.

12 Health and Social Care Information Centre. Hospital Episode Statistics. 2013. www.hesonline.nhs.uk Date last accessed: November 8, 2013.

\title{
HIV and multidrug-resistant tuberculosis: overlapping epidemics
}

\author{
To the Editor:
}

People infected with Mycobacterium tuberculosis and HIV are much more likely to develop active tuberculosis (TB) than people with M. tuberculosis but without HIV [1]. Patients infected with multidrugresistant (MDR)-TB (defined as resistance to at least rifampicin and isoniazid, the two most powerful antiTB drugs) require longer, more expensive treatment regimens than drug-susceptible $\mathrm{TB}$, with poorer treatment success $[2,3]$. Therefore, MDR-TB poses a major challenge to the control of $\mathrm{TB}$, with an estimated global disease incidence in 2012 of $\sim 450000$ cases (95\% CI 300 000-600 000) [4]. Although HIV is a powerful risk factor for all forms of TB and institutional outbreaks of MDR-TB among people living with HIV have been reported [5], population-level data on the association between HIV infection and MDR-TB are limited.

We explored the relationship between HIV infection and MDR-TB disease using data reported by member states to the World Health Organization (WHO) within the context of the Global Project on Anti-TB Drug Resistance Surveillance. The data were aggregated numbers of cases reported from either drug resistance surveys or continuous surveillance systems. Such surveys are epidemiological studies designed to measure drug resistance among a representative sample of notified pulmonary TB patients. Continuous surveillance is based on routine drug susceptibility testing of all bacteriologically confirmed TB patients. Subnational level data that were not representative of the entire country were excluded from the analysis, except for the Russian Federation and Ukraine, which are high MDR-TB burden countries for which high quality national level data were not available. The data included in the analysis met the criteria for data quality and national representativeness provided in detail elsewhere $[4,6]$. The laboratory methods used for diagnosis were endorsed by WHO.

For each country, the relationship between HIV infection and MDR-TB disease was investigated by logistic regression to calculate odds ratios and 95\% confidence intervals, using Stata (version 12; StataCorp, College Station, TX, USA). For countries with data from multiple years, robust standard errors accounted for within-country time dependencies. In order to minimise bias, data for a given year were excluded if $\leqslant 25 \%$ of reported TB patients had a documented HIV test result. 41 countries, accounting for $25 \%$ of the estimated global MDR-TB burden in 2012, met the inclusion criteria for $\geqslant 1$ year. Most were high-income countries and/or in the European Region. Only four countries were in the African region, which accounted for $75 \%$ of the global number of HIV-positive TB cases in 2012 [4]. Of these 41 countries, odds ratios could be calculated for 24 countries; the other 17 countries reported no HIV-positive MDR-TB cases (table 1). Complete HIV and MDR-TB data were available for 104781 TB patients from 1997-2012.

For 11 of the 24 countries for which the analysis was performed, HIV-positive TB patients had a significantly higher odds $(\mathrm{p}<0.05)$ of MDR-TB disease than HIV-negative TB patients (table 1$)$. Seven of these countries were in eastern Europe and central Asia: Estonia, Kazakhstan, Latvia, the Republic of Moldova, the Russian Federation, the Ukraine and Uzbekistan. For almost all of these 11 countries, the prevalence of MDR-TB among newly diagnosed TB cases (table 1) was higher than the estimated global average of 3.6\% (95\% CI 2.1-5.1\%) in 2012 [4]. Although the odds ratio was highest in Kuwait, only low numbers of HIV-positive cases were reported. HIV-positive TB patients in the USA had a lower odds of MDR-TB disease than HIV-negative patients.

As data were included if $\geqslant 75 \%$ of reported TB patients had a documented HIV test, the possibility of bias due to missing data cannot be ruled out. Although a positive association between HIV infection and MDRTB disease was demonstrated in less than half of the countries, a recent systematic review and meta-analysis found an odds of MDR-TB in HIV-positive patients that was 1.24 times (95\% CI 1.04-1.43) higher than in 